\title{
Finite element modelling of deformation behaviour in incremental sheet forming of aluminium alloy
}

\author{
Tsung-Han Huang ${ }^{1}$ and Cho-Pei Jiang ${ }^{2, a}$ \\ ${ }^{1}$ Institute of Mechanical and Electro-mechanical Engineering, National Formosa University, Yunlin, \\ Taiwan \\ ${ }^{2}$ Department of Power Mechanical Engineering, National Formosa University, Yunlin, Taiwan
}

\begin{abstract}
In this paper, the finite element method (FEM) is used to study the incremental sheet forming process of pyramidal shape. The material used is aluminium alloy 5052. The tool, a hemispherical ball-head with a diameter $(\mathrm{d}=4 \mathrm{~mm})$ made of HSS tool steel, is used to press down on the sheet metal causing locally plastic deformation. The comparison between spiral tool path, spiral-step tool path and z-level tool path is carried out. Moreover, the final thickness distribution is investigated. The results indicate that the minimal thickness can be found on the corner of wall angle in SPIF process. Under the same step over, spiral-step tool path can obtain the deepest depth for pyramidal shape. The maximum formability for successful forming of the pyramidal shape with depth $60 \mathrm{~mm}$ is wall angles $65^{\circ}$.
\end{abstract}

\section{Introduction}

Single Point Incremental Forming (SPIF) involves a local and progressive pressing out of the desired shape on a clamped sheet by a round-headed forming tool which follows a continuous path. SPIF offers full flexibility because the use of dedicated tooling isn't required. However, one disadvantage is that the operation time is high. Furthermore, SPIF may still be of use for low volume series and in combination with other forming processes to produce part details.

Thickness reduction is a phenomenon common to any sheet metal forming process that leads to the occurrence of crack and fracture of the resulting shape. In the numerical study of SPIF, the deformation behaviour such as thickness distribution and the mechanical properties of sheet metal on a pyramidal shape was investigated [1,2]. These reports present the thickness distribution in the final product is not uniform. Since tool path defines the component geometry, the different tool path with different incremental steps may affect the formability. Spiral tool path and z-level tool path are proposed to test formability for truncated cones [3]. Experimental results show that inclination angle and incremental step of the tool path affect the deformability.

\footnotetext{
${ }^{a}$ Corresponding author: cpjiang@nfu.edu.tw
}

This is an Open Access article distributed under the terms of the Creative Commons Attribution License 4.0, which permits unrestricted use, distribution, and reproduction in any medium, provided the original work is properly cited. 
Table 1. Composition of Al5052.

\begin{tabular}{|l|l|l|l|l|l|l|l|}
\hline Al & $\mathbf{M g}$ & $\mathbf{C r}$ & $\mathbf{C u}$ & $\mathbf{F e}$ & $\mathbf{M n}$ & $\mathbf{S i}$ & $\mathbf{Z n}$ \\
\hline Balance & 2.5 & 0.3 & 0.05 & 0.25 & 0.05 & 0.05 & 0.05 \\
\hline
\end{tabular}

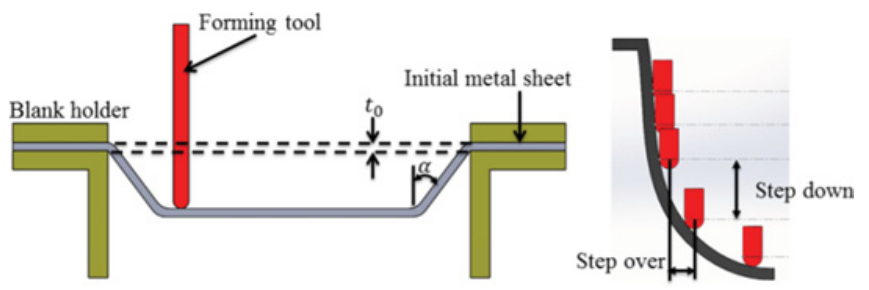

Figure 1. Details of incremental forming.

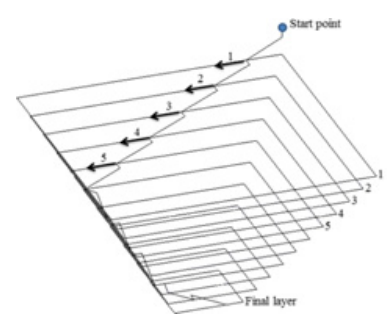

(a)

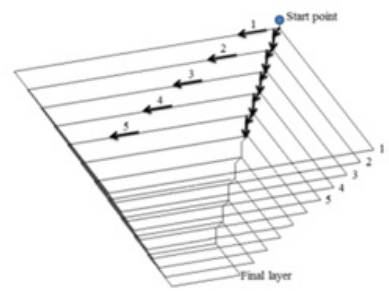

(b)

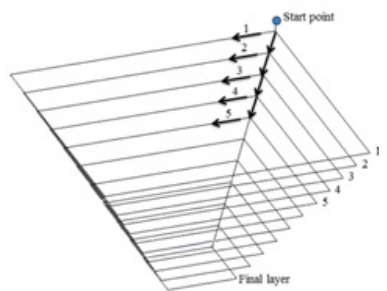

(c)

Figure 2. Tool paths for pyramidal shape: (a) spiral tool path, (b) spiral-step tool path and (c) z-level tool path.

In this paper, the finite element method is used to study the single point incremental forming process of pyramidal shape. The effect of spiral tool path, spiral-step tool path and z-level tool path on pyramidal depth and minimal thickness is investigated.

\section{Materials and method}

\subsection{Forming strategy}

The proposed part is a quadrangular pyramid. The initial dimension of Al5052 is $86 \times 86 \times 0.6 \mathrm{~mm}$. The base dimension of pyramidal shape is $60 \times 60 \mathrm{~mm}$ and the height is $60 \mathrm{~mm}$. The maximal inclination angle is a variation as indicator of forming limit. The composition of this material is shown in Table 1 .

\subsubsection{Tool path}

Figure 1 shows the details of single point incremental forming and the illustration of incremental steps. Step over is an adjustable parameter quantity to generate the tool path. For the investigated pyramidal with the variation inclination angle, step over is used to generate three kinds of tool paths (spiral, spiralstep and z-level). The spiral-step tool path in Fig. 2(a) is continuous with an incremental descent of the tool distributed over the entire surface of a part. Wall angle can be calculated by the trigonometric relationship of step over and step down Fig. 2(b). 


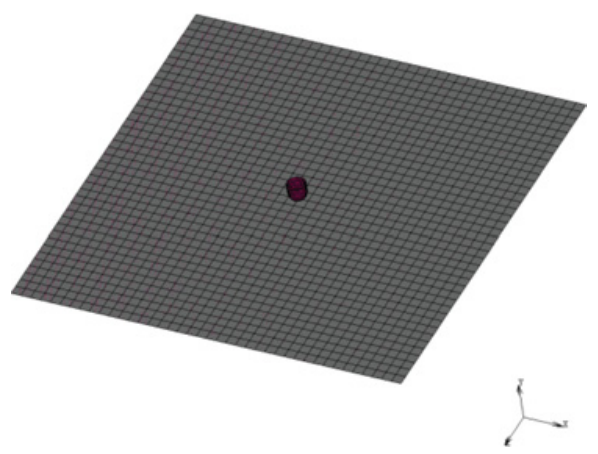

Figure 3. Numerical model used for incremental sheet metal forming process.

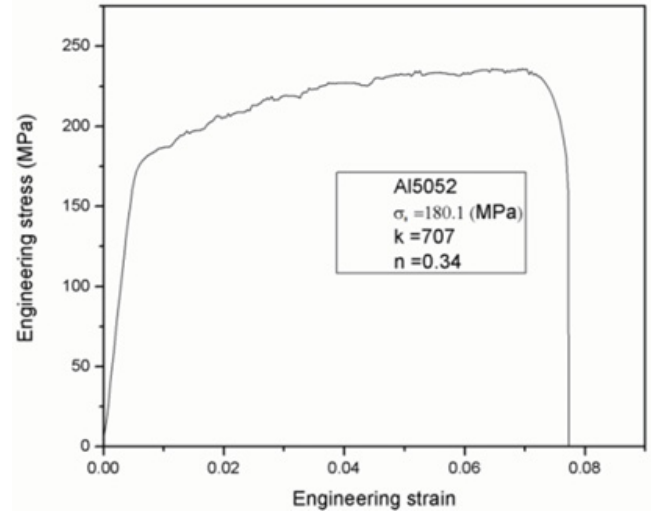

(a)

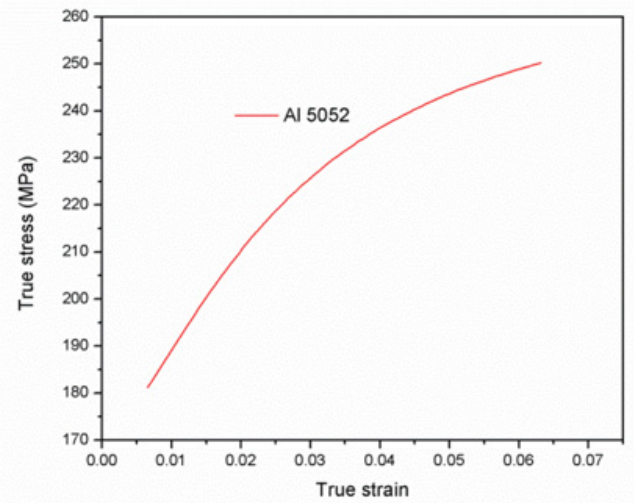

(b)

Figure 4. Engineering stress-strain curve for 5052 aluminium alloy sheet with $0.6 \mathrm{~mm}$.

\subsection{Finite element model}

A set of forming tool and sheet used to simulate the deformation behavior with different tool path in SPIF process as shown in Fig. 3. The sheet material is considered to be isotropic, homogeneous and incompressible. The sheet is meshed by shell element with four nodes and assumed deformable. Nodes on all edges of the sheet are constrained as fixed displacement and rotation during the forming process. The rigid tool shape is a cylinder with hemispherical head whose diameter is $4 \mathrm{~mm}$. The friction between sheet and tool is modelled using the Coulomb friction with a friction coefficient value of 0.13 .

\subsection{Material properties}

The material is underwent the tensile test and obtained the engineering stress-strain curve as shown in Fig. 4(a). The power law equation (1) describes the material behaviour in incremental sheet forming:

$$
\sigma=\mathrm{k} \varepsilon^{n}
$$

where $\sigma$ is the equivalent stress, $\varepsilon$ denotes the equivalent strain, $\mathrm{k}$ is the strength coefficient and $\mathrm{n}$ is the strain-hardening exponent. Therefore, the curve of true stress-strain can be shown in Fig. 4(b). The required mechanical properties of Al-5052 used in simulation are given in Table 2. 


\section{MATEC Web of Conferences}

Table 2. Mechanical properties for 5052 aluminium alloy sheet.

\begin{tabular}{|c|c|c|c|c|}
\hline $\mathrm{k}$ & $\mathrm{n}$ & $\mathrm{E}(\mathrm{GPa})$ & $\sigma_{0}$ & $\mathrm{v}$ \\
\hline 707 & 0.34 & 35.2 & 180.1 & 0.3 \\
\hline
\end{tabular}

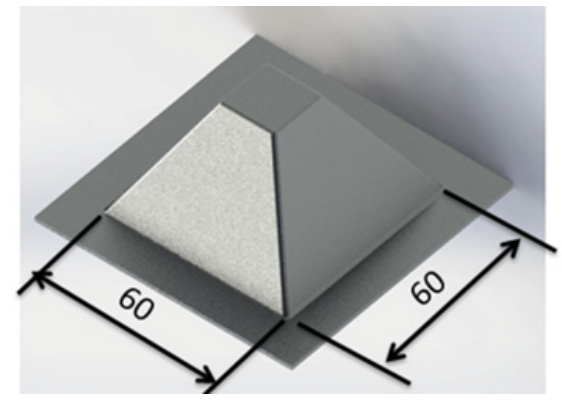

Figure 5. Benchmark- a pyramidal shape.

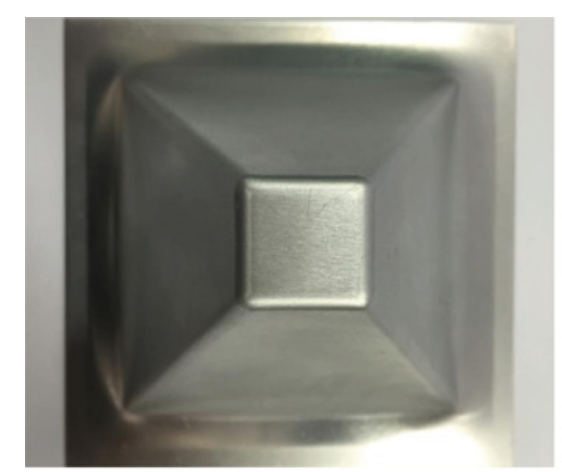

(a)

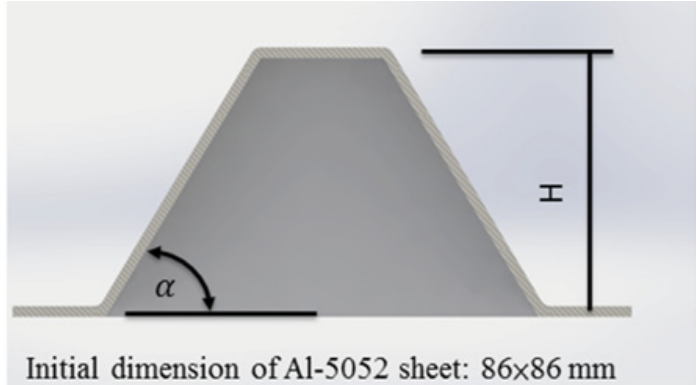

Initial dimension of $\mathrm{Al}-5052$ sheet: $86 \times 86 \mathrm{~mm}$

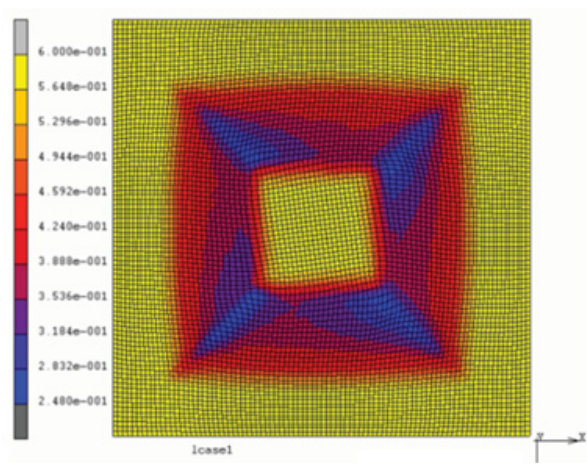

(b)

Figure 6. The complete benchmark shape: experimental (a) and simulation (b) results.

\section{Results and discussion}

In SPIF, the formability is higher than the deep drawing and stamping. Research literatures report that wall angle can be a formability indicator. In this study, the maximum wall angle is used as indicator to evaluate the formability of Al-5052 sheet with $0.6 \mathrm{~mm}$ thickness considering the effect of three types of tool path. A pyramidal shape is proposed as the benchmark as show in Fig. 5.

\subsection{Effect of tool path on formability}

Figure 6(a) shows the complete pyramidal shape with base dimension of $60 \times 60 \mathrm{~mm}$ and the height of $60 \mathrm{~mm}$. The tool movement is spiral-step as show in Fig. 2(b) and step-down is $0.5 \mathrm{~mm}$. The final wall angle is $69^{\circ}$. The simulation result presents the same shape as shown Fig. 6(b). The wall angle is the same and no fracture occurs after the tool completes the movement. Hence, the thickness of wall is compared as shown in Fig. 7. The minimal thickness which is $0.03 \mathrm{~mm}$ can be found on the corner of wall angle. 


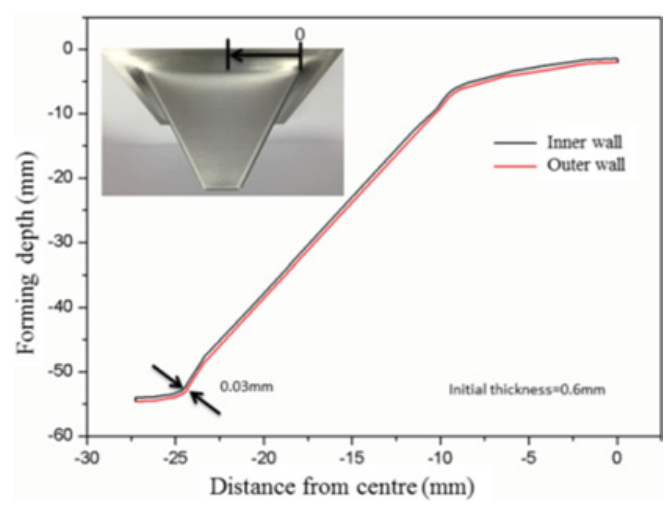

Figure 7. The comparison of wall thickness between experiment and simulation.

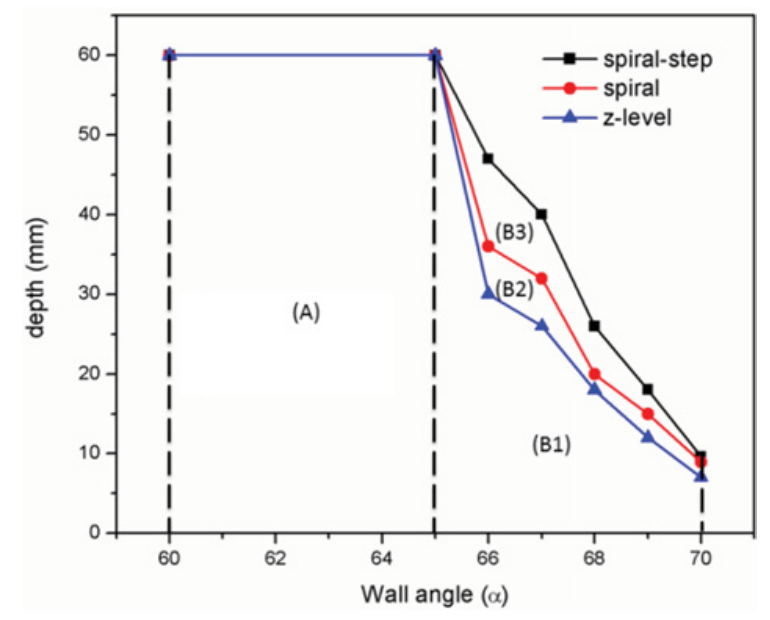

Figure 8. Influence of spiral, spiral-step and z-level tool paths on formability vs. wall angle $(\alpha)$.

\subsection{Forming limit diagram}

In the first test, the step over was set to be a constant value $0.5 \mathrm{~mm}$ for all tool paths. A series of pyramidal shapes were simulated with wall angle $60^{\circ}, 65^{\circ}, 66^{\circ}, 67^{\circ}, 68^{\circ}, 69^{\circ}$ and $70^{\circ}$ to evaluate the formability. Figure 8 presents the simulation results. Under the same step over, no noticeable different on formability between spiral, spiral-step and z-level tool paths. As can be seen, the maximum formability for successful forming of the pyramidal shape with depth $60 \mathrm{~mm}$ is wall angles $65^{\circ}$. A safety formability region is identified in area $\mathrm{A}$ for taking the required pyramid height into consideration. The area B3 has larger safety formability zone for spiral-step tool path than those for others. Therefore, spiral-step tool path can obtain the deepest depth for pyramidal shape with $0.5 \mathrm{~mm}$ step over size. 


\section{MATEC Web of Conferences}

\section{Conclusions}

Deformation behaviour of Al-5052 sheet in an incremental process for various tool paths is investigated. Formability is evaluated to understand the forming mechanism. Some conclusion can be drawn as follows:

(1) The minimal thickness can be found on the corner of wall angle in SPIF process.

(2) Under the same step over, spiral-step tool path can obtain the deepest depth for pyramidal shape.

(3) The maximum formability for successful forming of the pyramidal shape with depth $60 \mathrm{~mm}$ is wall angles $65^{\circ}$.

Funding from the National Science Council of Taiwan for this project, NSC101-2628-E-150-002-MY3, is gratefully acknowledged.

\section{References}

[1] S. Dejardin, S. Thibaud, J.C. Gelin, G. Michel, J. Mater. Process Tech. 210, 363-369 (2010)

[2] W.C. Emmens, G. Sebastiani, A.H. Van den Boogaard, J. Mater. Process Tech. 210, 981-997 (2010)

[3] Z. Liu, Y. Li, P.A. Meehan, Int. J. Pre. Eng. Man. 14, 1891-1899 (2013) 